\title{
Swift J1734.5-3027: a new long Type-I X-ray bursting source
}

\author{
E. Bozzo ${ }^{1}$, P. Romano ${ }^{2}$, M. Falanga ${ }^{3,4}$, C. Ferrigno ${ }^{1}$, A. Papitto ${ }^{5}$, and H. A. Krimm ${ }^{6,7}$
}

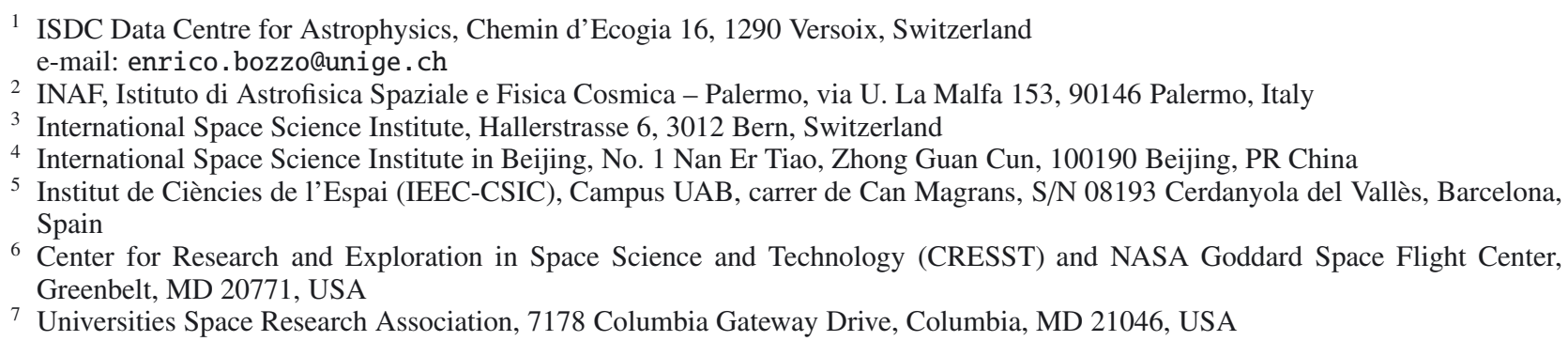

Received 21 March 2015 / Accepted 22 May 2015

\begin{abstract}
Swift J1734.5-3027 is a hard X-ray transient discovered by Swift while undergoing an outburst in September 2013. Archival observations showed that this source underwent a previous episode of enhanced X-ray activity in 2013 May-June. In this paper we report on the analysis of all X-ray data collected during the outburst in 2013 September, the first that could be intensively followed up by several X-ray facilities. Our dataset includes INTEGRAL, Swift, and XMM-Newton observations. From the timing and spectral analysis of these observations, we show that a long Type-I X-ray burst took place during the source outburst, making Swift J1734.5-3027 a new member of the class of bursting neutron star low-mass X-ray binaries. The burst lasted for about $1.9 \mathrm{ks}$ and reached a peak flux of $(6.0 \pm 1.8) \times 10^{-8} \mathrm{erg} \mathrm{cm}^{-2} \mathrm{~s}^{-1}$ in the $0.5-100 \mathrm{keV}$ energy range. The estimated burst fluence in the same energy range is $(1.10 \pm 0.10) \times 10^{-5} \mathrm{erg} \mathrm{cm}^{-2}$. By assuming that a photospheric radius expansion took place during the first $\sim 200 \mathrm{~s}$ of the burst and that the accreted material was predominantly composed by He, we derived a distance to the source of $7.2 \pm 1.5 \mathrm{kpc}$.
\end{abstract}

Key words. X-rays: binaries - X-rays: individuals: Swift J1734.5-3027

\section{Introduction}

Swift J1734.5-3027 is a hard X-ray transient discovered by Swift on 2013 September 1 (Malesani et al. 2013). At discovery, the source spectrum could be reasonably well described in the Swift/BAT energy band $(15-150 \mathrm{keV})$ by either a blackbody model with temperature $k T=2.5 \pm 0.4 \mathrm{keV}$, or a power-law model with photon index $\Gamma=5.8 \pm 0.7$. A fluence of $(1.6 \pm 0.3) \times$ $10^{-7} \mathrm{erg} \mathrm{cm}^{-2}$ was recorded during the initial $\sim 27 \mathrm{~s}$ of the BAT trigger (Kennea et al. 2013). These spectral characteristics led Kennea et al. (2013) to suggest that Swift J1734.5-3027 was a new superbursting X-ray transient. The source outburst was also observed with the instruments on-board INTEGRAL, providing additional information on the source broad-band spectrum (Kuulkers et al. 2013). An archival search into previous Swift observations performed in the directions of Swift J1734.5-3027 showed that the source underwent a likely previous outburst in 2013 May-June (La Parola et al. 2013). Unfortunately, in that occasion no follow-up observations were performed.

In this paper, we report on all X-ray data collected from Swift J1734.5-3027 during its outburst in 2013 September. In addition to the data from the INTEGRAL and Swift monitoring, we also analyze a target of opportunity (ToO) observation that we obtained with XMM-Newton on 2013 September 8. From the timing and spectral analysis of these data, we show that Swift J1734.5-3027 is a new member of the transient bursting neutron star low-mass X-ray binaries (NSLMXBs) and that the BAT discovery of the source occurred during a long Type-I $\mathrm{X}$-ray burst (rather than a superburst as suggested previously).

\section{INTEGRAL data}

Swift J1734.5-3027 was detected by INTEGRAL for the first time during the observations performed toward the Galactic bulge in the satellite revolution 1329, i.e., about half a day before the BAT discovery (from 56535.85950 MJD to 56536.01338 MJD; see Table 1). It remained within the field of view (FoV) of the instruments on-board INTEGRAL until satellite revolution 1348 (from 56592.54664 MJD to 56593.31612 MJD), when the window of seasonal visibility toward the Galactic center closed. We analyzed all INTEGRAL data by using version 10.1 of the Off-line Scientific Analysis software (OSA) distributed by the ISDC (Courvoisier et al. 2003). INTEGRAL observations are divided into science windows (SCWs), i.e., pointings with typical durations of $\sim 2-3 \mathrm{ks}$. To limit the ISGRI calibration uncertainties (Lebrun et al. 2003), we made use of all public SCWs from the Galactic bulge, Scutum/Sagittarium, and Perseus/Norma monitoring programs during which the source was located to within $12 \mathrm{deg}$ from the center of the IBIS FoV (Ubertini et al. 2003). We also included in our dataset the observations of the Galactic center and 4U 172834 for which our group was awarded data rights in revolutions 1329-1348. A summary of the total exposure-time available in each revolution is provided in Table 1 for IBIS/ISGRI and the two JEM-X telescopes (Lund et al. 2003). We extracted the mosaics and spectra in each revolution for the two JEM-X and ISGRI. All JEM-X spectra were extracted by using the standard 16-channel response matrix, while a customized 37 energy bin response matrix was created for ISGRI in order to optimize 
Table 1. Log of the INTEGRAL data used in this paper.

\begin{tabular}{lllllll}
\hline \hline Rev. & \multicolumn{2}{c}{ Observing time } & \multicolumn{2}{c}{ Exposure time } & \multicolumn{2}{c}{ Flux $^{a}$} \\
& START & STOP & ISGRI & JEM-X & ISGRI & JEM-X $^{b}$ \\
& (MJD) & (MJD) & $(\mathrm{ks})$ & $(\mathrm{ks})$ & $(20-70 \mathrm{keV})$ & $(3-35 \mathrm{keV})$ \\
\hline 1329 & 56535.85950 & 56536.01338 & 8.6 & 4.9 & $13.0 \pm 2.0$ & $<12.0$ \\
1330 & 56538.69428 & 56539.63178 & 73.3 & 8.0 & $19.7 \pm 1.0$ & $16.1 \pm 4.0$ \\
1331 & 56541.84263 & 56542.66694 & 63.2 & 3.2 & $<20.5$ & $<3.5$ \\
1332 & 56544.85712 & 56545.84130 & 74.0 & 7.7 & $<2.7$ & $<9.8$ \\
1333 & 56548.63922 & 56548.79324 & 10.9 & 4.7 & $<3.8$ & $<12.0$ \\
1334 & 56552.33453 & 56553.07995 & 59.3 & 7.3 & $<3.1$ & $<12.5$ \\
1335 & 56553.63791 & 56554.41490 & 58.0 & 10.8 & $<3.0$ & $<8.7$ \\
1336 & 56556.62811 & 56559.26417 & 71.3 & 14.6 & $<2.3$ & $<7.2$ \\
1337 & 56559.80640 & 56560.39413 & 45.4 & 10.9 & $<2.9$ & $<8.2$ \\
1339 & 56565.59969 & 56568.21845 & 199.7 & 23.9 & $8.8 \pm 0.4$ & $7.2 \pm 2.0$ \\
1340 & 56569.43604 & 56571.05972 & 105.2 & 25.8 & $7.6 \pm 0.7$ & $<5.8$ \\
1341 & 56572.63094 & 56574.06932 & 112.8 & 24.5 & $5.1 \pm 0.7$ & $<5.6$ \\
1343 & 56577.59515 & 56578.53990 & 69.0 & 13.6 & $9.8 \pm 0.9$ & $<7.6$ \\
1344 & 56580.71753 & 56582.17201 & 109.7 & 25.7 & $7.5 \pm 0.6$ & $<5.5$ \\
1345 & 56586.02326 & 56586.58723 & 45.5 & 7.6 & $14.0 \pm 1.0$ & $15.3 \pm 3.6$ \\
1346 & 56586.73297 & 56589.00642 & 110.4 & 20.1 & $8.7 \pm 0.7$ & $10.8 \pm 2.2$ \\
1347 & 56589.72509 & 56590.51950 & 63.3 & 12.0 & $6.6 \pm 0.8$ & $19.0 \pm 2.8$ \\
1348 & 56592.54664 & 56593.31612 & 60.8 & 5.8 & $8.3 \pm 1.1$ & $22.2 \pm 5.0$ \\
\hline
\end{tabular}

Notes. We report the effective exposure times, detection significance and estimated fluxes for ISGRI, JEM-X1, and JEM-X2 in all revolutions considered for the data analysis. The detection significances are reported for ISGRI (JEM-X1,2) in the 20-40 keV (3-10 keV) energy band. We considered a detection reliable in ISGRI and JEM-X only $>6 \sigma$ and $>4 \sigma$, respectively. ${ }^{(a)}$ The flux in mCrab has been estimated by comparing the source count-rate measured from the mosaic to the count-rate from the Crab in the same energy band. The count-rate from the Crab has been measured by extracting ISGRI, JEM-X1, and JEM-X2 mosaics of the public calibration observations performed in satellite revolutions 1342 (i.e., the calibration observations performed during the outburst of Swift J1734.5-3027). From these data we measured a Crab count-rate of $206.1 \pm 0.2 \mathrm{cts} \mathrm{s}^{-1}$ in ISGRI $\left(20-70 \mathrm{keV}\right.$ energy band) and $119.4 \pm 0.6 \mathrm{cts} \mathrm{s}^{-1}(134.5 \pm 0.7)$ for JEM-X2 (JEM-X1) in the $3-35 \mathrm{keV}$ energy band. (b) We used JEM-X2 as a reference. Lower significance detections have been considered only to derive upper limits on the source X-ray emission (all upper limits are at $3 \sigma$ c.l.).

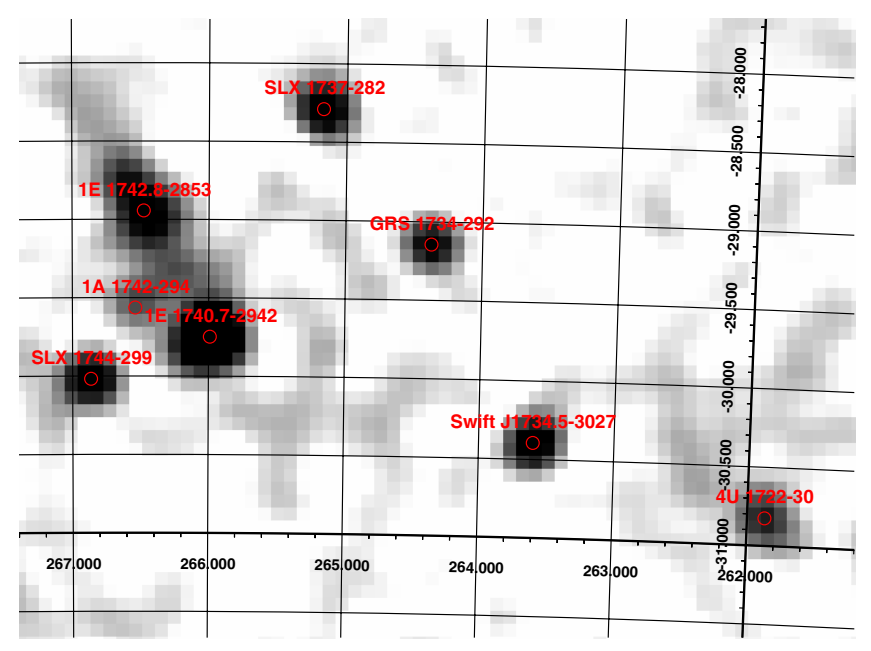

Fig. 1. INTEGRAL IBIS/ISGRI mosaiked image centered around the position of Swift J1734.5-3027. We used all available ISGRI data mentioned in Table 1 to build the mosaic $(20-70 \mathrm{keV})$. The source is detected in this mosaic with a significance of $34 \sigma$.

the signal-to-noise ratio $(\mathrm{S} / \mathrm{N})$ in the energy range $(20-50 \mathrm{keV})$. Only in revolution 1329, was the ISGRI spectrum extracted with a reduced energy binning (8 channels), as the source was relatively faint (see Sect. 3). JEM-X lightcurves with a time resolution of $2 \mathrm{~s}$ were extracted from all observations to search for Type-I X-ray bursts, but none was found. We did not perform other timing analyses of the INTEGRAL data as the source was too faint to extract meaningful power spectra. In Fig. 1 we show the ISGRI mosaic realized by using all available data.

\section{Swift data}

\subsection{XRT and BAT}

Swift/XRT observations were carried out from 56536.38578 MJD to 56763.42292 MJD (see Table 4). XRT data were collected in both windowed-timing (WT) and photon-counting (PC) mode and analyzed by using standard procedures within FTOOLS (v6.16). The XRT data were processed with the XRTPIPELINE (v.0.13.1) and the latest calibration files available (20140730). PC source events were extracted in annular regions with outer radii ranging between 20 to 30 pixels (1 pixel 2.36" $)$ depending on source intensity. When required, the PC data were corrected for pile-up. We determined the size of the point spread function (PSF) core affected by pile-up through a comparison between the observed and nominal PSF (see Vaughan et al. 2006). This sets the inner radius of the extraction region. WT source events were extracted in a circular region (20 pix radius), with the exception of observation 00569022000 during which the data were also affected by pileup; in the latter case an annular region (radii 2 and 20 pix) was adopted (see Romano et al. 2006). Background events were extracted in a circular regions with a radius of 70 pix for PC data and 20 pix for WT data. The XRTLCCORR task was then used to account pile-up, PSF losses, and vignetting, in the light curves. All light curves were background subtracted. For our timing analysis we converted the event arrival times to the solar system barycenter with BARYCORR. For our spectral analysis, we extracted events in the same regions as those adopted for the light-curve creation; ancillary response files were generated with XRTMKARF, to account for different extraction regions, vignetting and PSF corrections. The data were rebinned with 


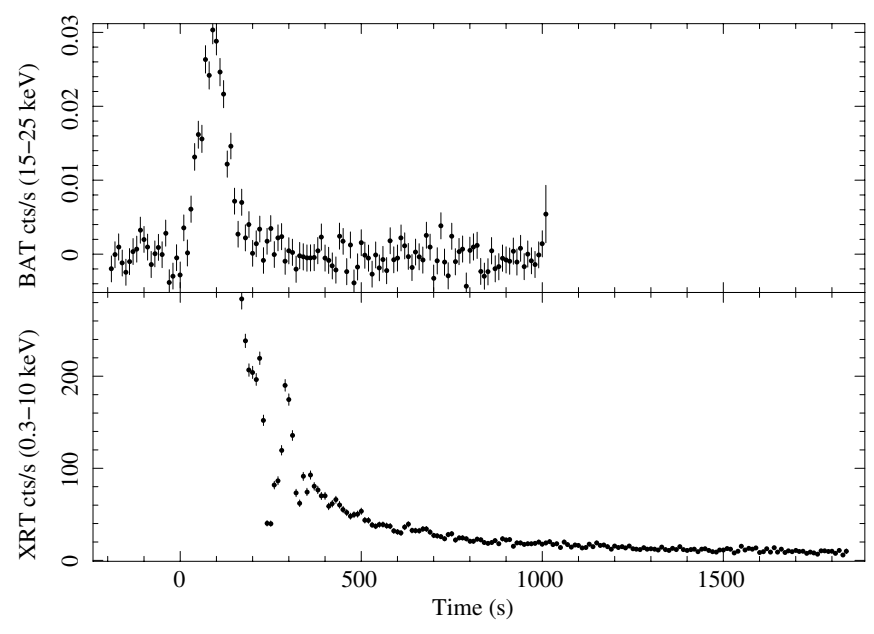

Fig. 2. BAT lightcurve corresponding to the on-board trigger at the onset of the outburst from Swift J1734.5-3027 (upper panel), together with the lightcurve extracted from the first available XRT observation performed after the Swift automatic slew in the direction of the source (ID. 00569022000). The time bin of both curves is $10 \mathrm{~s}$. The start time $(t=0)$ is set at $56536.38458 \mathrm{MJD}$.

a minimum of 20 count per energy bin to allow $\chi^{2}$ fitting. We also made use of BAT data corresponding to the on-board trigger of the source. The BAT lightcurve and spectra were extracted by using the BATGRBPRODUCT tool available within HEASOFT V. 6.16.

The best determined source position with XRT is found at: $\operatorname{RA}(\mathrm{J} 2000)=17^{\mathrm{h}} 34^{\mathrm{m}} 24^{\mathrm{s}} .18, \operatorname{Dec}(\mathrm{J} 2000)=-30^{\circ} 23^{\prime} 53^{\prime \prime} .0$ with an associated uncertainty of 1."4 (90\% c.1.). This was obtained by using the online Swift "User Object" tools ${ }^{1}$, which uses field stars in the UVOT to determine the spacecraft attitude (Evans et al. 2009).

The BAT lightcurve corresponding to the on-board trigger is shown in Fig. 2, together with the first XRT follow-up observation performed after the automatic slew of the spacecraft (first orbit of the observation ID. 00569022000). The XRT lightcurve covering the entire source outburst is shown in Fig. 3. We note that the source rose in flux rapidly at the onset of the event and then decayed slowly up to 56545 MJD. From 56545 MJD to 56571 MJD the source remained in a relatively low flux level before rising again in X-rays on 56572 MJD. During the lowluminosity interval, an XMM-Newton ToO observation was carried out (see Sect. 4).

We extracted 19 XRT spectra for the first orbit of the XRT observation ID. 00569022000 collected in WT mode (total exposure time 1668 s; see Fig. 2). The integration time of these spectra ranged from a few up to $300 \mathrm{~s}$, depending on the source flux during the burst decay (see Fig. 4). All 19 spectra could be well fit together by using an absorbed blackbody model $\left(\chi_{\text {red }}^{2} /\right.$ d.o.f. $=$ $1.10 / 1863)$. We left the absorption column density free to vary in the fit but tied the value of this parameter to be the same for all spectra $\left(N_{\mathrm{H}}=(0.84 \pm 0.03) \times 10^{22} \mathrm{~cm}^{-2}\right)$. For comparison, we tested that a fit with an absorbed power-law model gave an unacceptable result $\left(\chi_{\text {red }}^{2} /\right.$ d.o.f. $\left.=1.41 / 1863\right)$. The results of this time resolved analysis are summarized in Fig. 4. We also show in Fig. 5 the quasi simultaneous spectrum obtained from the BAT trigger (exposure time $113 \mathrm{~s}$ centered around the peak of the event) and the XRT spectrum extracted during the first $60 \mathrm{~s}$ of the observation ID. 00569022000 (overlapping with the last part

\footnotetext{
1 http://www.swift.ac.uk/user_objects/
}

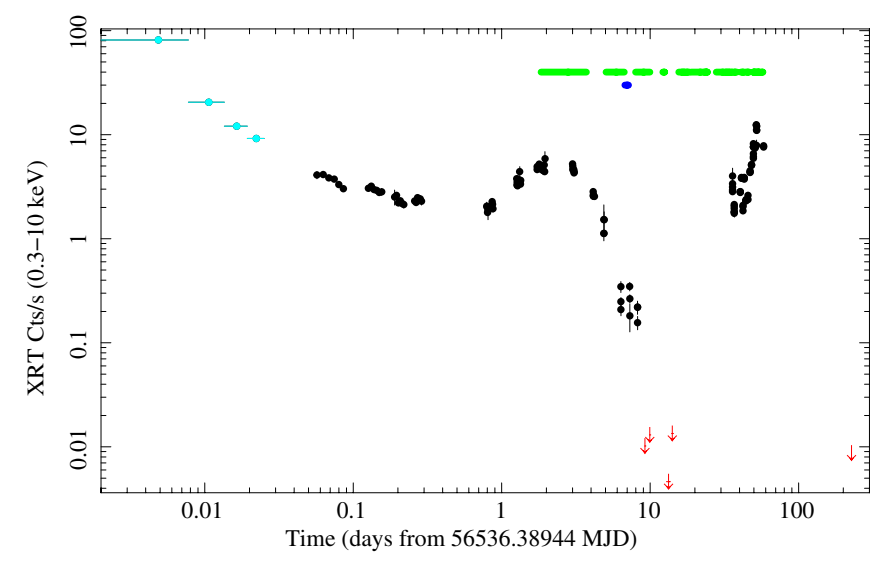

Fig. 3. XRT lightcurve of the entire outburst observed by Swift J1734.53027 (the start time is set at 56536.38458 MJD as in Fig. 2 and the bin time is $500 \mathrm{~s}$ ). The points in cyan represents the long Type-I X-ray burst. The red arrows show the XRT observations in which the source was not detected, providing an upper limit on the source count-rate. The green bands indicate the coverage of the outburst provided by INTEGRAL (data of revolution 1329 are not shown, as they were collected before the Swift discovery). We also indicated with a blue bar the time of the XMM-Newton ToO observation.

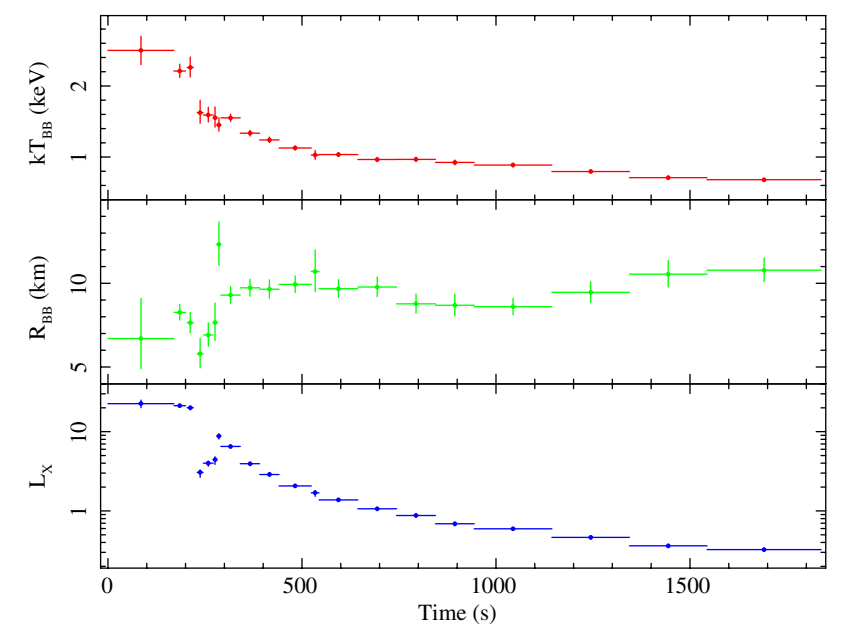

Fig. 4. Results of the time resolved spectral analysis carried out during the first part of the outburst decay. All spectra were fit with an absorbed blackbody model. We show in the three panels, from top to bottom, the blackbody temperature, radius, and the X-ray luminosity in units of $10^{37} \mathrm{erg} \mathrm{s}^{-1}$ for an assumed distance of $7.2 \mathrm{kpc}$ (see Sect. 5.1). The luminosity has been computed from the source flux extrapolated in the $0.5-100 \mathrm{keV}$ energy band (i.e., virtually bolometric for a blackbody spectral model with a temperature of $\sim 3 \mathrm{keV}$ ) and corrected for absorption. The first point on the left is obtained from the fit to the BAT spectrum (in this case we also extrapolated the source flux in the 0.5-100 keV energy band; see Sect. 5.1).

of the burst decay as detected by BAT; see Fig. 2). By fitting only the BAT spectrum extracted from the $t=0$ in Fig. 2 until the beginning of the first XRT observation (exposure time $170 \mathrm{~s}$ ), we obtained a blackbody temperature of $k T=2.5 \pm 0.2 \mathrm{keV}$, a radius of $R_{\mathrm{BB}}=6.7_{-1.8}^{+2.4} d_{7.2 \mathrm{kpc}} \mathrm{km}$, and a flux of $(5.1 \pm 0.5) \times$ $10^{-9} \mathrm{erg} \mathrm{cm}^{-2} \mathrm{~s}^{-1}$ in the $15-50 \mathrm{keV}$ energy range (corresponding to an extrapolated flux of $(3.6 \pm 0.4) \times 10^{-8} \mathrm{erg} \mathrm{cm}^{-2} \mathrm{~s}^{-1}$ in the $0.5-100 \mathrm{keV}$ energy range). The BAT spectrum extracted during the peak of the event (exposure time $1 \mathrm{~s}$ ) gave instead $k T=3.0_{-0.7}^{+1.0}, R_{\mathrm{BB}}=6_{-6}^{+10} d_{7.2 \mathrm{kpc}} \mathrm{km}$, and a $15-50 \mathrm{keV}$ flux of $(1.3 \pm 0.4) \times 10^{-8} \mathrm{erg} \mathrm{cm}^{-2} \mathrm{~s}^{-1}$. The latter would correspond to 


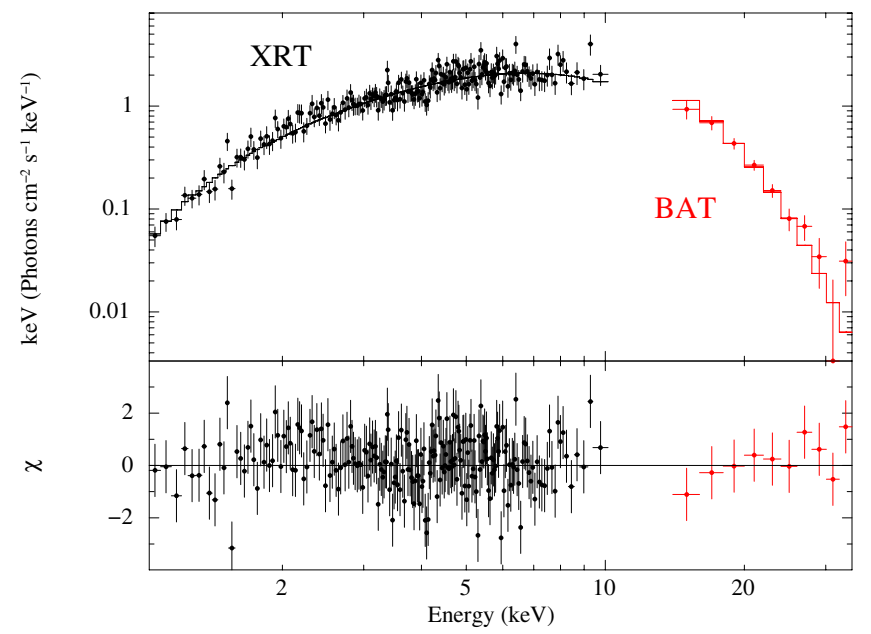

Fig. 5. Quasi-simultaneous XRT and BAT spectrum of the source at the onset of the outburst. The BAT spectrum is integrated for $113 \mathrm{~s}$ around the peak of the event, while the XRT spectrum is extracted from the first $30 \mathrm{~s}$ of the observation ID. 00569022000 (overlapping with the last part of the decaying BAT lightcurve; see Fig. 2). The best fit model is obtained with an absorbed blackbody model $\left(\chi_{\text {red }}^{2} /\right.$ d.o.f. $\left.=1.02 / 233\right)$. The fit gave $N_{\mathrm{H}}=(0.7 \pm 0.1) \times 10^{22} \mathrm{~cm}^{-2}, k T=2.4 \pm 0.1 \mathrm{keV}$, and $R_{\mathrm{BB}}=$ $7.3 \pm 0.5 d_{7.2 \mathrm{kpc}} \mathrm{km}$. The residuals from the fit are shown in the bottom panel. The estimated $0.5-50 \mathrm{keV}$ X-ray flux is $4.5 \times 10^{-8} \mathrm{erg} \mathrm{cm}^{-2} \mathrm{~s}^{-1}$ (not corrected for absorption).

Table 2. Parameters of the long Type-I X-ray burst.

\begin{tabular}{ll}
\hline \hline$F_{\text {peak }}{ }^{a}\left(\mathrm{erg} \mathrm{cm}^{-2} \mathrm{~s}^{-1}\right)$ & $(6.0 \pm 1.8) \times 10^{-8}$ \\
$f_{\mathrm{b}}^{b}\left(\mathrm{erg} \mathrm{cm}^{-2}\right)$ & $(1.10 \pm 0.10) \times 10^{-5}$ \\
$\tau_{1} \equiv f_{\mathrm{b}} / F_{\text {peak }}(\mathrm{s})$ & $178_{-53}^{+98}$ \\
$\gamma \equiv F_{\text {pers }}{ }^{c} / F_{\text {peak }}$ & $(8.7 \pm 4.2) \times 10^{-3}$ \\
\hline
\end{tabular}

Notes. ${ }^{(a)}$ Unabsorbed flux $(0.5-100 \mathrm{keV}) .{ }^{(b)}$ Fluence $(0.5-100 \mathrm{keV})$. (c) Unabsorbed persistent flux $F_{\text {pers }}=(5.2 \pm 0.2) \times 10^{-10} \mathrm{erg} \mathrm{cm}^{-2} \mathrm{~s}^{-1}$ $(0.5-100 \mathrm{keV})$, as extrapolated from the closest XRT observation after the burst, i.e., ID. 00569022001.

an extrapolated flux of $(6.0 \pm 1.8) \times 10^{-8} \mathrm{erg} \mathrm{cm}^{-2} \mathrm{~s}^{-1}$ in the energy range $0.5-100 \mathrm{keV}$. Based on the spectral analysis and the flux evolution, we suggest in Sect. 5 that the event recorded by XRT during the observation ID. 00569022000 is a long Type-I $\mathrm{X}$-ray burst of a NSLMXB. In Table 2 we report the most relevant properties of the burst.

The source X-ray spectra extracted from all the XRT data collected after the first orbit of the observation ID. 00569022000 could not be well fit by using an absorbed blackbody model. In all these cases, an absorbed power-law model provided a better description of the data (see Table 4). The power-law spectral index varied along the outburst from 1.6 to $\sim 3$, and there is some hint of a decrease in the absorption column density in the direction of the source from $1.5 \times 10^{22} \mathrm{~cm}^{-2}$ to $0.8 \times 10^{22} \mathrm{~cm}^{-2}$ during the latest available observations.

We searched all XRT data for possible coherent pulsations and/or quasi-periodic oscillations, but no significant feature was found. We obtained the most stringent upper limit on the detection of coherent modulations by using the XRT WT data from the observation ID. 00569022000. In this case we estimated a $3 \sigma$ c.l. upper limit of $5.6 \%$ on the pulsed fraction of pulsations in frequency range $0.1-281 \mathrm{~Hz}$ (the pulsed fraction is defined as in Vaughan et al. 1994).

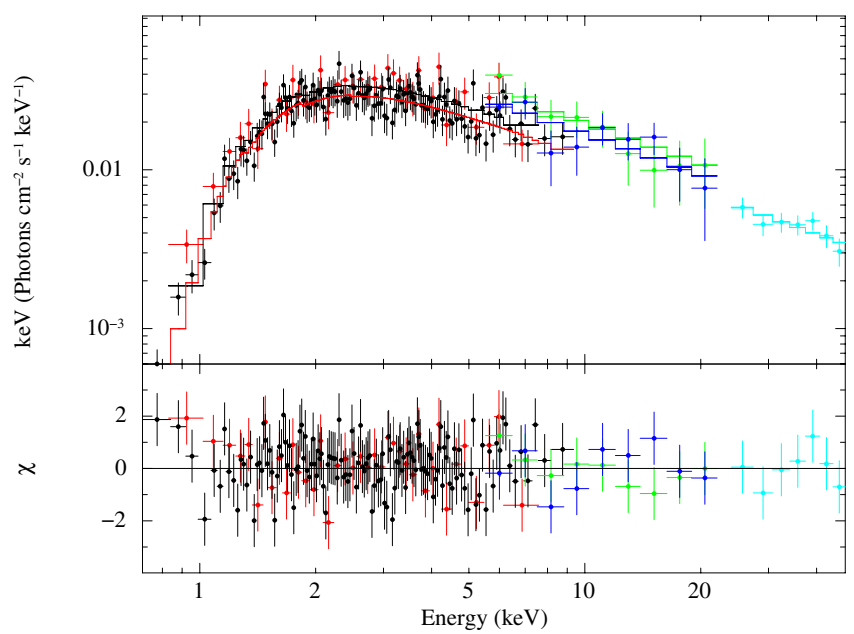

Fig. 6. Broad-band spectrum of Swift J1734.5-3027 obtained by using the XRT WT (red) and PC (black) spectra from the observation ID. 00569022006, together with the ISGRI (cyan) and JEM-X (green for JEM-X1 and blue for JEM-X2) data from revolution 1330. The best fit is obtained by using a simple absorbed power-law model. The residuals from the fit are reported in the bottom panel.

We used the quasi-simultaneous Swift observation ID. 00569022006 and INTEGRAL data collected in revolution 1330 to study the broad-band spectrum of the source at the later stages of the outburst. We fit together the XRT spectra in PC and WT mode, with the ISGRI and JEM-X spectra. The best fit could be obtained by using a simple absorbed power-law model (see Fig. 6). Normalization constants were included in the fit to take into account the inter-calibrations of the instruments and the source variability (data were not strictly simultaneous; see Tables 1 and 4). Taking advantage of the other quasi-simultaneous data, we also performed a similar broadband spectral analysis by using: (a) INTEGRAL data from revolution 1345 and the Swift observation ID. 00032930017; (b) INTEGRAL data from revolution 1346 and the Swift observation ID. 00032930019; (c) INTEGRAL data from revolution 1348 and the Swift observation ID. 00032930021. In all cases a simple absorbed power-law provided a good fit to the data. All the results are reported in Table 3.

All these spectra showed that the broad-band emission from the source was significantly harder during the later stages of the event compared to the peak of the outburst observed by BAT (that we interpreted as a long Type-I X-ray burst; see Sect. 5). To further confirm these findings, we extracted the ISGRI spectrum of the source from rev. 1329. This is the only INTEGRAL detection of the source before the BAT discovery. Although the source was relatively faint in the ISGRI energy band during rev. 1329, a sufficiently high $\mathrm{S} / \mathrm{N}$ spectrum could be extracted by using reduced energy binning ( 8 channels). A fit to this spectrum gave a power-law photon index of $2.3 \pm 0.7$ and a $20-50 \mathrm{keV}$ flux of $1.5 \times 10^{-10} \mathrm{erg} \mathrm{cm}^{-2} \mathrm{~s}^{-1}$.

For completeness, we also reported in Fig. 7 the long-term BAT lightcurve of the source as retrieved from the BAT Transient Monitor web-page ${ }^{2}$ (Krimm et al. 2013). The lightcurve starts on MJD 56352 (i.e., 2013 March 1). The outburst reported in this paper and the previous detection of Swift J1734.5-3027 in May-June 2013 are clearly visible. The latter detection was also discussed by La Parola et al. (2013). These authors confirmed that the BAT spectral index at that time was consistent with that

2 http://swift.gsfc.nasa.gov/results/transients/weak/SwiftJ1734.5-3027/ 
E. Bozzo et al.: Swift J1734.5-3027: a new long Type-I X-ray bursting source

Table 3. Combined INTEGRAL and Swift broad-band spectral analysis of Swift J1734.5-3027 (see also Sect. 3.1 for details).

\begin{tabular}{llllllllll}
\hline \hline INTEGRAL rev. & XRT obs. & $\begin{array}{l}N_{\mathrm{H}} \\
\left(10^{22} \mathrm{~cm}^{-2}\right)\end{array}$ & $\Gamma$ & $C_{\mathrm{XRT} T}$ & $C_{\text {JEM-X1 }}$ & $C_{\text {JEM-X2 }}$ & $C_{\mathrm{ISGRI}}$ & $F_{20-50 \mathrm{keV}}{ }^{a}$ & $\chi_{\text {red }}^{2} /$ d.o.f. \\
\hline 1330 & 00569022006 & $1.30 \pm 0.10$ & $1.90 \pm 0.10$ & $0.87 \pm 0.06$ & $1.4 \pm 0.3$ & $1.2 \pm 0.2$ & $0.9 \pm 0.2$ & 7.7 & $0.87 / 198$ \\
1345 & 00032930017 & $0.88 \pm 0.06$ & $2.26 \pm 0.08$ & - & $1.0 \pm 0.3$ & $1.5 \pm 0.3$ & $1.6 \pm 0.4$ & 6.0 & $0.96 / 250$ \\
1346 & 00032930019 & $0.94 \pm 0.05$ & $2.34 \pm 0.07$ & - & - & $1.1 \pm 0.2$ & $0.9 \pm 0.2$ & 6.9 & $0.88 / 306$ \\
1348 & 00032930021 & $0.75 \pm 0.05$ & $2.26 \pm 0.08$ & - & $2.2 \pm 0.8$ & $3.2 \pm 0.7$ & $1.6 \pm 0.4$ & 6.1 & $1.0 / 244$ \\
\hline
\end{tabular}

Notes. $C_{\mathrm{XRT}}, C_{\mathrm{JEM}-\mathrm{X} 1}, C_{\mathrm{JEM}-\mathrm{X} 2}$, and $C_{\mathrm{ISGRI}}$ are the normalization constants of the different instruments compared to the XRT spectrum (for which the constant was fixed to unity in the fit). (a) The flux is not corrected for absorption and given in units of $10^{-10} \mathrm{erg} \mathrm{cm}^{-2} \mathrm{~s}^{-1}$.

Table 4. Log of all SwiftXRT observations use in this work.

\begin{tabular}{|c|c|c|c|c|c|c|c|c|}
\hline Sequence & Obs/Mode & $\begin{array}{l}\text { Start time (UT) } \\
\text { (MJD) }\end{array}$ & $\begin{array}{l}\text { End time (UT) } \\
\text { (MJD) }\end{array}$ & $\begin{array}{l}\text { Exposure } \\
\text { (s) }\end{array}$ & $\begin{array}{l}N_{\mathrm{H}} \\
\left(10^{22} \mathrm{~cm}^{-2}\right)\end{array}$ & $\Gamma$ & $\begin{array}{l}F_{0.5-10 \mathrm{keV}^{a}} \\
\left(10^{-10} \mathrm{erg} \mathrm{cm}^{-2} \mathrm{~s}^{-1}\right)\end{array}$ & $\chi_{\text {red }}^{2} /$ d.o.f. \\
\hline $00569022000^{b}$ & XRT/WT & 56536.38578 & 56536.40508 & 1668 & & & & \\
\hline 00569022000 & XRT/PC & 56536.44215 & 56536.58503 & 5581 & $1.49 \pm 0.10$ & $2.39 \pm 0.10$ & $1.6 \pm 0.6$ & $0.92 / 211^{c}$ \\
\hline 00569022001 & XRT/WT & 56536.58612 & 56536.64248 & 395 & $1.2 \pm 0.2$ & $2.3 \pm 0.3$ & $1.26 \pm 0.09$ & $1.11 / 145^{c}$ \\
\hline 00569022001 & XRT/PC & 56536.58623 & 56536.67200 & 3698 & $1.5 \pm 0.2$ & $2.4 \pm 0.2$ & $1.14 \pm 0.06$ & $1.11 / 145^{c}$ \\
\hline 00569022002 & XRT/WT & 56537.17595 & 56537.37689 & 1412 & $1.4 \pm 0.2$ & $2.3 \pm 0.2$ & $1.11 \pm 0.106$ & $0.91 / 186^{c}$ \\
\hline 00569022002 & XRT/PC & 56537.17604 & 56537.25757 & 2565 & $1.4 \pm 0.2$ & $2.1 \pm 0.2$ & $1.1 \pm 0.1$ & $0.91 / 186^{c}$ \\
\hline $00569022003^{d}$ & XRT/WT & 56537.64302 & 56537.71031 & 153 & - & - & - & - \\
\hline 00569022003 & XRT/PC & 56537.64434 & 56537.72605 & 3814 & $1.24 \pm 0.13$ & $1.93 \pm 0.11$ & $2.00 \pm 0.07$ & $0.97 / 152$ \\
\hline $00569022004^{d}$ & XRT/WT & 56538.11015 & 56538.33109 & 324 & - & - & - & - \\
\hline 00569022004 & XRT/PC & 56538.11176 & 56538.33635 & 3638 & $1.01 \pm 0.09$ & $1.62 \pm 0.10$ & $3.00 \pm 0.10$ & $1.05 / 185$ \\
\hline 00569022006 & XRT/WT & 56539.38610 & 56539.45124 & 159 & $1.06 \pm 0.29$ & $1.7 \pm 0.3$ & $3.3 \pm 0.3$ & $0.91 / 184^{c}$ \\
\hline 00569022006 & XRT/PC & 56539.38740 & 56539.46243 & 2164 & $1.36 \pm 0.13$ & $1.88 \pm 0.13$ & $2.81 \pm 0.12$ & $0.91 / 184^{c}$ \\
\hline 00569022007 & XRT/WT & 56540.51553 & 56540.59720 & 1890 & $1.33 \pm 0.14$ & $2.25 \pm 0.13$ & $1.10 \pm 0.06$ & $1.06 / 162$ \\
\hline 00569022008 & XRT/WT & 56541.26421 & 56541.27637 & 1024 & $1.6 \pm 0.3$ & $3.0 \pm 0.3$ & $0.34 \pm 0.03$ & $1.04 / 40$ \\
\hline 00569022009 & XRT/WT & 56542.73167 & 56542.74373 & 1021 & $1.4_{-1.3}^{+4.8}$ & $2.5_{-1.5}^{+3.3}$ & $0.03_{-0.02}^{+0.01}$ & $1.32 / 5$ \\
\hline $00032930001^{e}$ & XRT/WT & 56543.66528 & 56543.67847 & 925 & $1.4^{-1.5}$ & $2.5^{-1.5}$ & $\sim 0.01$ & - \\
\hline $00032930002^{e}$ & XRT/WT & 56544.60000 & 56544.61319 & 1068 & 1.4 & 2.5 & $\sim 0.005$ & - \\
\hline $00032930003^{f}$ & XRT/PC & 56545.60069 & 56545.61389 & 1096 & 1.6 & 2.5 & $<0.005$ & - \\
\hline $00032930004^{f}$ & XRT/PC & 56546.32569 & 56546.33889 & 973 & 1.6 & 2.5 & $<0.006$ & - \\
\hline $00032930005^{f}$ & XRT/PC & 56549.46944 & 56549.88472 & 3814 & 1.6 & 2.5 & $<0.002$ & - \\
\hline $00032930006^{f}$ & XRT/PC & 56550.39653 & 56550.54861 & 3936 & 1.6 & 2.5 & $<0.006$ & - \\
\hline 00032980001 & XRT/PC & 56572.28104 & 56572.30564 & 1973 & $1.6 \pm 0.2$ & $2.3 \pm 0.2$ & $1.68 \pm 0.07$ & $1.14 / 76$ \\
\hline 00032980002 & XRT/PC & 56573.22292 & 56573.29167 & 1878 & $1.5 \pm 0.2$ & $2.4 \pm 0.2$ & $1.00 \pm 0.06$ & $0.82 / 45$ \\
\hline 00032930007 & XRT/WT & 56576.83264 & 56576.84583 & 947 & $1.1 \pm 0.2$ & $2.2 \pm 0.2$ & $1.15 \pm 0.08$ & $1.03 / 84$ \\
\hline 00032930008 & XRT/WT & 56577.63889 & 56577.65208 & 980 & $1.27 \pm 0.14$ & $2.10 \pm 0.14$ & $1.82 \pm 0.08$ & $0.92 / 127$ \\
\hline 00032930009 & XRT/WT & 56578.55903 & 56578.57222 & 944 & $1.6 \pm 0.4$ & $2.4 \pm 0.4$ & $0.89 \pm 0.07$ & $0.85 / 31$ \\
\hline 00032930010 & XRT/WT & 56579.50069 & 56579.51458 & 1126 & $1.26 \pm 0.14$ & $2.06 \pm 0.12$ & $1.87 \pm 0.08$ & $1.05 / 152$ \\
\hline 00032930011 & XRT/WT & 56580.63472 & 56580.64931 & 1044 & $1.2 \pm 0.2$ & $2.2 \pm 0.2$ & $1.02 \pm 0.06$ & $0.89 / 93$ \\
\hline 00032930013 & XRT/WT & 56582.09583 & 56582.10833 & 913 & $1.3 \pm 0.2$ & $2.2 \pm 0.2$ & $1.17 \pm 0.07$ & $0.98 / 85$ \\
\hline 00032930014 & XRT/WT & 56583.43056 & 56583.44444 & 1064 & $1.15 \pm 0.11$ & $2.04 \pm 0.11$ & $2.21 \pm 0.07$ & $0.88 / 159$ \\
\hline 00032930015 & XRT/WT & 56584.56458 & 56584.57847 & 1025 & $0.93 \pm 0.09$ & $2.00 \pm 0.10$ & $2.40 \pm 0.07$ & $1.08 / 171$ \\
\hline 00032930016 & XRT/WT & 56585.97014 & 56585.98333 & 961 & $0.91 \pm 0.08$ & $2.28 \pm 0.10$ & $2.80 \pm 0.10$ & $0.86 / 176$ \\
\hline 00032930017 & XRT/WT & 56586.04097 & 56586.05417 & 960 & $0.88 \pm 0.06$ & $2.27 \pm 0.08$ & $3.23 \pm 0.11$ & $0.98 / 222$ \\
\hline 00032930018 & XRT/WT & 56587.44236 & 56587.45556 & 526 & $0.74 \pm 0.07$ & $2.14 \pm 0.11$ & $3.2_{-0.2}^{+0.1}$ & $0.99 / 142$ \\
\hline 00032930019 & XRT/WT & 56588.37708 & 56588.51667 & 1049 & $0.94 \pm 0.05$ & $2.36 \pm 0.07$ & $4.63 \pm 0.10$ & $0.89 / 292$ \\
\hline 00032930021 & XRT/WT & 56594.45208 & 56594.46458 & 946 & $0.76 \pm 0.05$ & $2.27 \pm 0.08$ & $3.00 \pm 0.08$ & $1.06 / 221$ \\
\hline $00033239001^{f}$ & XRT/PC & 56763.28194 & 56763.42292 & 2021 & 0.76 & 2.27 & $<0.004$ & - \\
\hline
\end{tabular}

Notes. For each observation we also report the results of the spectral fit carried out by using a simple absorbed power-law model (phabs*pow in XSPEC). ${ }^{(a)}$ Observed flux (i.e., not corrected for absorption). ${ }^{(b)}$ Analyzed separately (see Sect. 3 for details). ${ }^{(c)}$ We fit the PC and WT spectrum together, leaving all parameters free to vary but obtaining a single $\chi_{\text {red }}^{2} /$ d.o.f. from the fit. ${ }^{(d)}$ Statistics was too poor to extract a meaningful spectrum. ${ }^{(e)}$ The source was detected but too faint for the XRT WT mode. A rough estimate of the source flux is given from the recorded count rate and assuming the spectral model of the previous observation with good statistics. ${ }^{(f)}$ The source was not detected and thus we used the spectral model of the preceding observation to derive a $3 \sigma$ upper limit on the source X-ray flux.

measured by ISGRI before the onset of the bright event recorded in September 2013 and during the latest stages of such event (see Sects. 1 and 3.1). Unfortunately, no pointed XRT observations were performed in May-June 2013.

\subsection{UVOT}

We also analyzed all available UVOT data that were collected simultaneously with the XRT observations. The analysis of the UVOT data was performed using the UVOTIMSUM and 


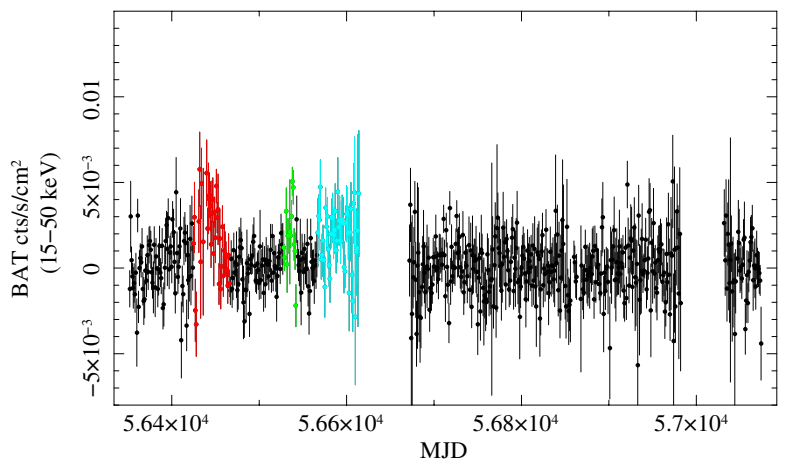

Fig. 7. Long-term BAT lightcurve of SwiftJ1734.5-3027 in the 15-50 keV energy range. The two outbursts of the source in May-June 2013 (red points) and September 2013 (green and cyan points) are clearly visible. For the outburst in September, we highlighted in green the initial part of the event up to the quiescent period around 56545 MJD. The cyan points mark the re-brightening of the source after the quiescent period up to 56614 MJD. Gaps in the BAT lightcurve are due to the source proximity to the Sun.

UVOTSOURCE tasks included in the FTOOLS software. The latter task performs aperture photometry on a single source based on user-supplied source and background regions ${ }^{3}$; we adopted circular regions of $5^{\prime \prime}$ and $10^{\prime \prime}$ for source and background, respectively. A faint source is found within the XRT error circle in the coadded UVOT $u$ images (total exposure $5977 \mathrm{~s}$ ). For this source we obtained a magnitude of $u=19.70 \pm 0.12 \mathrm{mag}$. The best estimated UVOT position for this object is at RA $(\mathrm{J} 2000)=$ $17^{\mathrm{h}} 34^{\mathrm{m}} 24^{\mathrm{s}} 33, \operatorname{Dec}(\mathrm{J} 2000)=-30^{\circ} 23^{\prime} 53^{\prime \prime} .2$ with an uncertainty of 0.43 (90\% c.l.). This is compatible at $2.4 \sigma$ c.l. with the XRT position reported in Sect. 3.1 (assuming the XRT uncertainty in the position follows a Rayleigh distribution; Evans et al. 2014). Only poorly constraining upper limits could be obtained on the source magnitudes in the other UVOT filters. Owing to the lack of any detection of changes in magnitude of the UVOT source during the outburst and the marginal compatibility between the UVOT and XRT position, we are currently unable to firmly associate the counterpart identified in this section with Swift J1734.5-3027.

\section{XMM-Newton data}

An XMM-Newton (Jansen et al. 2001) observation of Swift J1734.5-3027 was carried out on 2013 September 8 for a total exposure time of $32.3 \mathrm{ks}$ (from 56543.1404 MJD to 56543.5293 MJD). The EPIC-pn was operated in timing mode, the MOS1 in full frame and the MOS2 in small window. We reduced these data by using the SAS version 13.5 and the latest XMM-Newton calibrations files available ${ }^{4}$. The observation was not affected by intervals of high flaring background; we thus retained the entire exposure time available for the following analysis. As the source was found to be relatively faint (see later in this section) and centered on RAWX $=37$, the EPIC-pn lightcurve and spectra were extracted in the energy range $0.6-12 \mathrm{keV}$ from CCD columns 36 through 38 for the source and 3 through 15 for the background. For the two MOS cameras we used a circular source (background) extraction region with a radius of 600 (1800) pixels, corresponding to $30^{\prime \prime}\left(90^{\prime \prime}\right)$. The average count-rate of the source as recorded by the EPIC-pn was $(8.3 \pm 0.2) \times 10^{-2}$ cts s$^{-1}$, thus too low to perform any count-rate

\footnotetext{
3 http://heasarc.nasa.gov/ftools/caldb/help/uvotsource.html

$4 \mathrm{http} / / / \mathrm{xmm} 2$. esac.esa.int/external/ xmm_sw_cal/calib/index.shtml
}

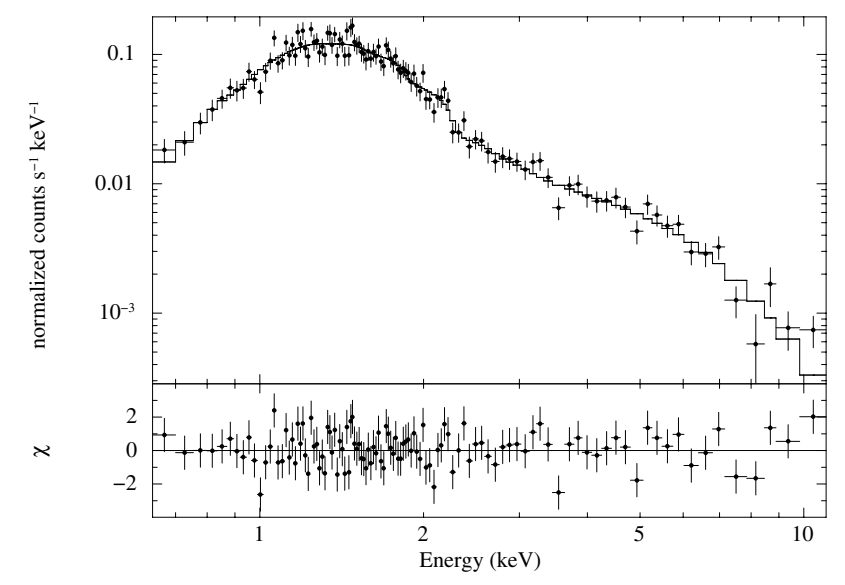

Fig. 8. XMM-Newton spectrum of Swift J1734.5-3027 obtained by summing up the Epic-pn, MOS1, and MOS2 data. The best fit model is obtained by using an absorbed power law plus a blackbody. The residuals from the fit are shown in the bottom panel.

and/or time resolved analysis. To improve the statistics, we used the task EPICSPECCOMBINE to combine all EPIC spectra and fit them together (see Fig. 8).

A fit to this spectrum with a simple absorbed power-law model gave an unacceptable result with $\chi_{\text {red }}^{2} /$ d.o.f. $=1.88 / 111$. The addition of a thermal blackbody component (BBODYRAD in XSPEC) significantly improved the fit $\left(\chi_{\text {red }}^{2} /\right.$ d.o.f. $\left.=1.07 / 109\right)$. We measured an absorption column density of $N_{\mathrm{H}}=(0.57 \pm$ $0.08) \times 10^{22} \mathrm{~cm}^{-2}$, a power-law photon index of $\Gamma=1.2 \pm 0.3$, a blackbody temperature of $k T=0.32 \pm 0.03 \mathrm{keV}$, and a radius of $R_{\mathrm{BB}}=\left(1.5_{-0.3}^{+0.5}\right) \times d_{7.2 \mathrm{kpc}} \mathrm{km}$. The average absorbed (unabsorbed) $0.5-10 \mathrm{keV}$ X-ray flux measured from the spectral fit is $5.5 \times 10^{-13} \mathrm{erg} \mathrm{cm}^{-2} \mathrm{~s}^{-1}\left(8.9 \times 10^{-13} \mathrm{erg} \mathrm{cm}^{-2} \mathrm{~s}^{-1}\right)$. This is consistent with the approximate measurement of the source flux obtained with XRT in the observations ID. 00032930001 and 00032930002 (see Table 4). The $N_{\mathrm{H}}$ measured by XMM-Newton suggests that the absorption column density in the direction of the source might slightly decrease not only during the latest stages of the outburst, but also during the low X-ray luminosity period (unfortunately during this period we could not obtain a reliable $N_{\mathrm{H}}$ measurement with XRT owing to the low statistics of the data; see Sect. 3).

We searched for possible coherent pulsations and/or quasiperiodic oscillations in the XMM-Newton data, but none was found (also owing to the very limited statistics). For coherent modulations, we estimated an upper limit of $13.5 \%$ at $3 \sigma$ c.l. on the pulsed fraction of pulsations in the frequency range $0.1-1500 \mathrm{~Hz}$.

For completeness, we checked for possible evidence of the soft thermal component detected by XMM-Newton in the XRT data collected close to 56543 MJD. In the XRT observations ID 00032930001-00032930006 the source was not detected. We thus considered the observation ID. 00569022007 , which was performed 2.4 days earlier than the XMM-Newton one and was characterized by a reasonably high statistics (see Table 4). The addition of a blackbody component to the source spectrum in this observation did not improve the fit. We thus fixed the temperature of this component to the one measured by XMM-Newton and determined a $90 \%$ c.l. upper limit on its normalization. We obtained $R_{\mathrm{BB}}<7 \mathrm{~km}$ (for an assumed distance of $7.2 \mathrm{kpc}$ ). This result suggests that the thermal emission revealed by XMM-Newton might have gone undetected during the earlier stages of the outburst due to the higher flux of the power-law component. We comment more on this result in Sect. 5. 


\section{Discussion}

In this paper we report on all X-ray data collected during the first followed up outburst of the source Swift J1734.5-3027, which also led to its discovery. Shortly after the onset of the outburst, the source displayed a long Type-I X-ray burst that we discuss in detail later in this section. This allows us to firmly classify the source as a new member of the bursting NSLMXBs.

As noted in Sect. 3, the source showed a remarkable decay in flux after the onset of the outburst up to 56539 MJD. At this epoch, the decay of the flux steepened dramatically and the source faded below the XRT detection limit on 56543 MJD (see Fig. 3). Such outburst profile is, in several respects, qualitatively similar to those observed from transient NSLMXBs. The outbursts of these systems typically last for a few weeks and are characterized by an initial exponential decay. At the later stages, a break is sometimes observed in the flux decay and a knee in the lightcurve marks the transition to a faster linear decaying phase that lasts until the source fades back into quiescence $^{5}$ (see, e.g., Gilfanov et al. 1998; Gierliński \& Zdziarski 2005; Falanga et al. 2005a,b, 2011, 2012). These outburst profiles are usually interpreted in terms of the disk instability model proposed by King \& Ritter (1998). These authors showed that the X-ray heating during the decay from an outburst causes the light curves of a transient LMXBs to exhibit an exponential decay if the X-ray luminosity is sufficiently high to maintain the outer disk radius hot, or a linear decay at lower luminosities. This model was successfully applied to a number of observed LMXB outbursts by Powell et al. (2007), who also showed that the luminosity at which the knee occurs in the lightcurve and the exponential timescale of the outburst decay can be used to estimate the source outer disk radius. Indeed, it is believed that the matter supply to the NS is effectively cut-off after the knee, when the irradiation of the outer disk radius diminishes and the disk enters a cool low-viscosity state.

The peculiarity of the Swift J1734.5-3027 outburst profile is the presence of an apparently quiescent period extending from 56545.6 MJD to at least 56550.5 MJD that preceded a new rebrightening. During the quiescent period the source remained in a flux level that was too low to be detectable within the short XRT exposures (see Table 4). Stacking together the XRT observations ID. 00032930003, 00032930004, 00032930005, and 00032930006 a $3 \sigma$ upper limit on the source count rate of 0.0049 cts s $^{-1}$ is obtained, corresponding to an X-ray luminosity of $1.6 \times 10^{33} \mathrm{erg} \mathrm{s}^{-1}$ (at $7.2 \mathrm{kpc}$, see below). As LMXBs in quiescence might have a luminosity as low as $\lesssim 10^{32} \mathrm{erg} \mathrm{s}^{-1}$, it is not possible to determine if Swift J1734.5-3027 really faded back into quiescence during this period or underwent an episode of very low mass accretion rate. Surprisingly, the source resumed its X-ray active state around 56572 MJD. There was no coverage available with XRT for the period 56550-56572 MJD, but the INTEGRAL data show that the high-intensity state of the source did not resume before 56565 MJD. The apparent low activity period in X-rays thus lasted about 15 days. At the beginning of the re-brightening phase the source recovered roughly the same $\mathrm{X}$-ray luminosity displayed immediately after the long Type-I $\mathrm{X}$-ray burst, and its broad-band X-ray spectrum was found to be fully compatible with that observed during most of the previous outburst. The mechanism that triggered this second episode of enhanced accretion is difficult to investigate due to the paucity of the data and the lack of coverage during the rise and the decay

\footnotetext{
In some cases, a linear decay in flux is observed directly after a stage in which the flux of the source is relatively stable for several weeks (see, e.g., Heinke et al. 2015; Campana et al. 2013).
}

from this renewed activity. The last available XRT observation of Swift J1734.5-3027 was carried out on 56763 MJD, i.e., about 169 days after the last detection of the source in the X-ray active phase, and thus we do not know precisely how long the second rebrightening phase lasted in time.

We note that a similar "double" outburst as described above was observed from the accreting millisecond X-ray pulsar (AMXP) IGR J00291+5934, which underwent two $\sim 10$ days long outbursts in 2008 separated by a quiescent period of $\sim 30$ days (Hartman et al. 2011). In this case, the first outburst followed the usual profile displaying a knee during the decay at a certain luminosity, while the second was characterized by a significantly slower rise and decay times. Owing to the presence of the second outburst, Hartman et al. (2011) questioned the applicability of the irradiated disk model to IGR J00291+5934. The main issue with this interpretation is that it predicts the disk around the NS to be heavily emptied after the first outburst and unable to power a second event without invoking an unlikely large variation in the mass flow rate from the companion star. The authors suggested that the knee during the decay of the first outburst could have been due to the onset of a propeller stage, during which accretion is (at least) partly inhibited due to the rapidly rotating NS magnetosphere (see, e.g., Illarionov \& Sunyaev 1975; Romanova et al. 2014, and references therein). The inhibition of accretion would leave enough material in the disk after the first outburst to fuel a second event within a few days. As the condition for the onset of a propeller effect depends strongly on the NS magnetic field strength and rotational period, it is difficult to test this scenario in the case of Swift J1734.53027 , for which neither the spin period nor its derivative are known (the NS magnetic field can be inferred from measurements of the spin period variations over time; see, e.g., Papitto et al. 2007, and references therein). Furthermore, it is worth noting that, although our understanding of the propeller regime is still far from complete, this effect is often involved to interpret more abrupt drops in luminosity (see, e.g., Ferrigno et al. 2014, for the recent case of the AMXP IGR J18245-2452).

The XMM-Newton spectrum of the source extracted during the faster portion of the first outburst decay (around 56543 MJD) could not be well described by a simple absorbed power-all model, at odds with all previously collected XRT spectra. The XMM-Newton data revealed the presence of a predominant thermal component, which properties are compatible with those expected for an emitting hot spot on the NS surface (see Sect. 4). As discussed in Sect. 4, we could not firmly rule out the presence of this thermal component in the XRT data; if present, the thermal emission could have been likely hidden by the dominant comptonization component (i.e., the power-law due to the accretion). We thus conclude that during the latest stages of the first outburst, accretion was strongly suppressed and the emission from the NS surface emerged at the soft X-rays $(\lesssim 2 \mathrm{keV})$. We are, however, currently unable to discriminate between a suppression of the accretion due to the disk turning into the low viscosity state or the onset of a mild propeller phase.

It is interesting to note that, at odds with a number of previously studied systems (Kuulkers et al. 2009), the long Type-I burst burst recorded by Swift J1734.5-3027 is unlikely to be the event triggering the overall outburst of the source. The INTEGRAL observations carried out in rev. 1329 already detected an enhanced X-ray activity from the source a few days before the onset of the long Type-I burst. This indicates that the NS was already accreting a substantial amount of matter at the time. We thus conclude that the outburst developed as a consequence of the disk instability, and it was the latter that paved the way 
for the long Type-I X-ray burst rather than the other way around. As bursts from Swift J1734.5-3027 were never detected before of the outburst in September 2013, it is unlikely that the source undergoes thermonuclear runaways during its quiescent state, as observed from the so-called burst-only sources (see, e.g., Cocchi et al. 2001). This would be expected according to the known stability of hydrogen burning via the pp-process or pycnonuclear reactions at low X-ray luminosities (Fushiki \& Lamb 1987). Assuming a quiescent luminosity for Swift J1734.5-3027 of $\sim 10^{33} \mathrm{erg} \mathrm{s}^{-1}$ (as estimated from the XRT observations and assuming a distance of $\sim 7.2 \mathrm{kpc}$; see Sect. 5.1.2), it would take $\sim 200$ years to the source to accumulate the necessary fuel on the neutron star surface to emit a burst with a characteristic energy release of $\sim 6.7 \times 10^{40} \mathrm{erg}$ (see Sect. 5.1.2). Therefore, it seems that Swift J1734.5-3027 gives rise to thermonuclear explosions only when it is accreting at luminosities $\gtrsim 10^{35}-10^{36} \mathrm{erg} \mathrm{s}^{-1}$ and is thus a new member of the faint transient NSLMXBs exhibiting pure helium runaways when in outburst.

\subsection{The long Type-I X-ray burst}

From the time resolved spectroscopy and flux evolution of the Swift data collected on 2013 September 1, we argued that the event triggering the BAT on that day was due to a long Type-I X-ray burst from Swift J1734.5-3027. The time and spectral properties of the event are strikingly similar to those of the long burst from the NSLMXB XTE J1701-407 that triggered the BAT on 2008 July 17 (Falanga et al. 2009). We discuss the physical properties of the long burst from Swift J1734.5-3027 in the next three sections and use our observational findings to derive constraints on the distance to the source and the details of the accretion process.

\subsubsection{Light curve and spectra}

Our analysis of the XRT and BAT data presented in this paper revealed a long Type-I X-ray burst from Swift J1734.5-3027. This is the first discovered burst from the source. In Fig. 4, we showed the BAT (upper panel) and XRT (lower panel) light curve of the long Type-I X-ray burst. We set the burst start time at 56536.38458 MJD, i.e., the time at which the BAT X-ray intensity of the source increased by $10 \%$ compared to its persistent intensity level. The XRT started observing the source about $170 \mathrm{~s}$ after the beginning of the burst detected by BAT. The BAT light curve exhibits a slow rise time 6 of $\approx 50 \mathrm{~s}$. The XRT decay time from the burst is best-fit by using two exponential functions with e-folding times of $\tau_{1}=110 \pm 8 \mathrm{~s}$ and $\tau_{2}=1019 \pm 85 \mathrm{~s}$, respectively. A single exponential function could not provide an acceptable fit to the data. We note that the value $\tau_{1} \equiv f_{\mathrm{b}} / F_{\text {peak }}=178_{-53}^{98}$ s reported in Table 2 is roughly consistent with the e-folding time estimated from the fit to the burst profile. Similar long Type-I X-ray bursts were so far recorded from the sources 2S 0918-549, SLX 1737-282, and XTE J1701-407 (in't Zand et al. 2005; Falanga et al. 2008; Linares et al. 2008; Falanga et al. 2009). To compare the burst from Swift J1734.5-3027 with these previous results and with the decay cooling model proposed by Cumming \& Macbeth (2004), we fitted the burst flux decay profile in Fig. 4 with a power-law, and found an index of $\Gamma=-1.77 \pm 0.02$ with a $\chi_{\text {red }}^{2} /$ d.o.f. $=1.5 / 17$. This is comparable to the results found in the other cases. The total duration of the burst, i.e., the time to evolve away from and return to the persistent state, was of $\approx 200 \mathrm{~s}$

\footnotetext{
6 The rise time is defined as the time spent between the start of the burst and the point at which the $90 \%$ of the peak burst intensity is reached.
}

and $\approx 30 \mathrm{~min}$ in the BAT (15-25 keV) and the XRT $(0.3-10 \mathrm{keV})$ lightcurves, respectively.

It is well known that X-ray bursts are produced by unstable burning of matter accreted on the surface of NSs and the X-ray emission released in this process can be described well as blackbody radiation with temperatures of a few $\mathrm{keV}$. The energydependent decay time of the burst is regulated by the cooling of the NS photosphere, which results in a gradual softening of the burst spectrum (see, e.g., Lewin et al. 1993; Strohmayer $\&$ Bildsten 2006). The time-resolved spectral analysis of the burst recorded from Swift J1734.5-3027 was carried out by using BAT and XRT data in the $15-25 \mathrm{keV}$ and $0.3-10 \mathrm{keV}$ bands, respectively. Burst spectra could be described well by a simple photoelectrically-absorbed blackbody model (we measured $N_{\mathrm{H}}=0.84 \times 10^{22} \mathrm{~cm}^{-2}$; see Table 2). The inferred blackbody temperature, apparent radius at $7.2 \mathrm{kpc}$ (see below), and bolometric luminosity are reported in Fig. 4 and in Table 2. The burst fluence has been calculated from the bolometric fluxes (these correspond to the observed $0.3-10 \mathrm{keV}$ XRT fluxes extrapolated to the $0.5-100 \mathrm{keV}$ energy range). The peak flux of the burst was derived from the BAT spectrum extrapolated to the $0.5-100 \mathrm{keV}$ energy range. All measured fluxes during the burst were extrapolated to the $0.5-100 \mathrm{keV}$ band by generating dummy responses (XSPEC v12.8.2). This is justifiable for the XRT data since the blackbody temperature is well inside the spectral energy coverage. During the main peak of the burst, the blackbody temperature reaches a maximum in the energy range $2-3 \mathrm{keV}$ (see, e.g., Falanga et al. 2008, and references therein), and thus the bolometric flux of the blackbody emission from the source is outside the BAT bandpass. However, as we detail below, this introduces uncertainties that are well within the estimated error on the bolometric flux measurements reported in Table 2 . The bestfit blackbody temperature measured from the BAT spectrum extracted during the peak of the burst was $k T=3.0_{-0.7}^{+1.0} \mathrm{keV}$. We fixed an upper and lower boundary blackbody temperature of 2.7 and $3.5 \mathrm{keV}$, respectively, the normalization remaining as a free parameter. The lower boundary for the temperature is chosen to be higher than the temperature measured during the XRT data collected close to the peak of the burst (see Fig. 4). For the upper boundary, we assumed that $k T \sim 3.5 \mathrm{keV}$ is a reasonably high value for the blackbody temperature at the peak of the event based on previous observations of long bursts from LMXBs (see, e.g., Molkov et al. 2005; Chenevez et al. 2006, 2007; Falanga et al. 2008, 2009). In the two cases, we found extrapolated fluxes of $4.4 \times 10^{-8} \mathrm{erg} \mathrm{cm}^{-2} \mathrm{~s}^{-1}$ and $7.0 \times 10^{-8} \mathrm{erg} \mathrm{cm}^{-2} \mathrm{~s}^{-1}$. These are well within the boundary of the peak flux uncertainties reported in Table 2. We note that a similar procedure was applied to extrapolate the peak flux of the burst in the case of XTE J1701-407 (Falanga et al. 2009).

\subsubsection{Source distance, persistent flux, and accretion rate}

In photospheric-radius expansion (PRE) bursts, the source distance can be determined based on the assumption that the bolometric peak luminosity is saturated at the Eddington limit ( $L_{\text {Edd }}$; e.g., Lewin et al. 1993; Kuulkers et al. 2003). During the PRE episode, the bolometric luminosity remains virtually constant at the Eddington value, while the high energy flux displays a typical double-peak profile and/or a delay in the rise time (e.g., Kuulkers et al. 2002; Galloway \& Cumming 2006; Falanga et al. 2007, 2008).

In the case of Swift J1734.5-3027, the BAT lightcurve shows a slow rise time of $\approx 50 \mathrm{~s}$, which is typically observed at the 
hard X-rays long Type-I helium bursts with PRE (e.g., Kuulkers et al. 2002; Molkov et al. 2005; Falanga et al. 2008). However, the XRT observation started about $170 \mathrm{~s}$ after the peak of the event, and thus we cannot firmly assess if this burst underwent a PRE. The time-resolved spectral analysis of the BAT data could not provide sufficient indications to solve this issue owing to the limited statistics (see Fig. 2). From the comparison between the burst from Swift J1734.5-3027 with other PRE bursts exhibiting a similar slow rise time at high energies, we assume in the following that Swift J1734.5-3027 underwent a PRE during the first $\approx 200 \mathrm{~s}$ of the event. This should correspond to the timescale that is needed by the photosphere to fall back onto the NS surface (see discussion in Falanga et al. 2009). Under these assumptions and considering a bolometric peak luminosity equal to the Eddington value for a He Type-I X-ray burst $\left(L_{\text {Edd }} \approx 3.8 \times 10^{38} \mathrm{erg} \mathrm{s}^{-1}\right.$, as empirically derived by Kuulkers et al. 2003), we obtain a source distance for Swift J1734.5-3027 of $d=7.2 \pm 1.5 \mathrm{kpc}$. We note that the source would be closer if the peak luminosity of the burst were lower than the pure-helium Eddington limit, or if a solar composition $\left(X_{0}=0.7\right)$ is assumed for the accreted material (in this case the source distance would be $\approx 6 \mathrm{kpc}$ ). In the following, we consider $d \approx 7.2 \mathrm{kpc}$ as a fiducial distance.

In the case of Swift J1734.5-3027, an estimate of the persistent emission immediately before the onset of the long Type-I burst was not available (see Sect. 3). We thus assume as the preburst persistent emission, the flux measured in the closest XRT observation (i.e., the PC data in observation ID. 00569022000). In these data, the X-ray emission from Swift J1734.5-3027 was found to be well described by a simple power-law model with $\Gamma \approx 2.4$. Assuming a distance of $7.2 \mathrm{kpc}$, the estimated long Type-I burst persistent unabsorbed flux in the energy range $0.5-100 \mathrm{keV}$ is thus $F_{\text {pers }} \approx 5.2 \times 10^{-10} \mathrm{erg} \mathrm{cm}^{-2} \mathrm{~s}^{-1}$, translating into a bolometric luminosity of $L_{\text {pers }} \approx 3.3 \times 10^{36} \mathrm{erg} \mathrm{cm}^{-2} \mathrm{~s}^{-1}$, i.e., $\approx 1.0 \% L_{\text {Edd }}$. We can estimate the local accretion rate per unit area as $L_{\text {pers }}=5.4 \pi R^{2} \dot{m}(G M / R) /(1+z)$, or

$$
\begin{aligned}
\dot{m} \sim & 2 \times 10^{3} \mathrm{~g} \mathrm{~cm}^{-2} \mathrm{~s}^{-1} \\
\times & \times\left(\frac{R}{11.2 \mathrm{~km}}\right)^{-1}\left(\frac{M}{1.4 M_{\odot}}\right)^{-1}\left(\frac{d}{7.2 \mathrm{kpc}}\right)^{2}\left(\frac{1+z}{1.26}\right) .
\end{aligned}
$$

A convenient unit of accretion rate is the Eddington rate. Here, we define the local Eddington accretion rate to be $\dot{m}_{\text {Edd }} \equiv$ $1.8 \times 10^{5} \mathrm{~g} \mathrm{~cm}^{-2} \mathrm{~s}^{-1}$, which corresponds to the local accretion rate of a NS of mass $M=1.4 M_{\odot}$ and radius $R=11.2 \mathrm{~km}$ that has a luminosity equal to the Eddington value $3.8 \times 10^{38} \mathrm{erg} \mathrm{s}^{-1}$ (Kuulkers et al. 2003). We thus conclude that $\dot{m} / \dot{m}_{\text {Edd }} \sim 1 \%$.

\subsubsection{Energy, ignition depth, and recurrence time of the burst}

The released energy measured during the long-burst allows us to estimate the ignition depth and derive predictions on the burst recurrence time. The measured fluence of the burst, $f_{\mathrm{b}}=$ $1.1 \times 10^{-5} \mathrm{erg} \mathrm{cm}^{-2}$, corresponds to a net burst energy release of $E_{\text {burst }}=4 \pi d^{2} f_{\mathrm{b}}=6.7 \times 10^{40} \mathrm{erg}(d / 7.2 \mathrm{kpc})^{2}$. The ignition depth is related to the energy release during the burst by the equation $E_{\text {burst }}=4 \pi R^{2} y_{\text {ign }} Q_{\text {nuc }} /(1+z)$, and thus

$$
\begin{aligned}
y_{\mathrm{ign}}= & 3.4 \times 10^{9} \mathrm{~g} \mathrm{~cm}^{-2}\left(\frac{d}{7.2 \mathrm{kpc}}\right)^{2} \\
& \times\left(\frac{Q_{\mathrm{nuc}}}{1.6 \mathrm{MeV} / \text { nucleon }}\right)^{-1}\left(\frac{R}{11.2 \mathrm{~km}}\right)^{-2}\left(\frac{1+z}{1.26}\right) .
\end{aligned}
$$

The value of $Q_{\text {nuc }} \approx 1.6 \mathrm{MeV} /$ nucleon corresponds to the nuclear-energy release per nucleon for complete burning of helium to iron group elements. Including hydrogen with a massweighted mean mass fraction $\langle X\rangle$ provides a value of $Q_{\text {nuc }} \approx$ $1.6+4\langle X\rangle \mathrm{MeV} /$ nucleon (Galloway et al. 2004), where we included losses due to neutrino emission following Fujimoto et al. (1987). For $\langle X\rangle=0.7$, the solar composition value, $Q_{\text {nuc }}=$ $4.4 \mathrm{MeV} /$ nucleon, and $y_{\text {ign }}=1.2 \times 10^{9} \mathrm{~g} \mathrm{~cm}^{-2}$. At an accretion rate of $2 \times 10^{3} \mathrm{~g} \mathrm{~cm}^{-2} \mathrm{~s}^{-1}$, the recurrence time corresponding to a column depth of $y_{\text {ign }}=3.4 \times 10^{9} \mathrm{~g} \mathrm{~cm}^{-2}$ (pure helium composition) is $\Delta t=\left(y_{\text {ign }} / \dot{m}\right)(1+z) \sim 24$ days, or for $y_{\text {ign }}=1.2 \times 10^{9} \mathrm{~g} \mathrm{~cm}^{-2}$ (solar composition) is $\Delta t \sim 9$ days (independent from the assumed distance).

The long Type-I burst, which was the first observed burst from Swift J1734.5-3027, occurred about half a day after the first INTEGRAL detection of the source in rev. 1329 (starting from $56535.85950 \mathrm{MJD}$ ). The outburst of the source was closely monitored only for the first 15 days, when the source first faded into a low luminosity state (see Sect. 3 and Fig. 3). The re-brightening of the source was observed starting from 56565.5999 MJD, thus leaving a gap in the outburst coverage of about 15 days during which we do not have information on the X-ray emission from the source. The last useful XRT observation of the source during the re-brightening was carried out up to $56594.46458 \mathrm{MJD}$, i.e., about 58 days from the detected long Type-I burst. According to our estimates above, the case $Q_{\text {nuc }} \approx 1.6 \mathrm{MeV} /$ nucleon would be slightly favoured by the data (as we did not observe a second Type-I X-ray burst), but the coverage of the outburst was far too sparse and limited in time to draw any firm conclusion.

Acknowledgements. E.B. thanks the New York University at Abu Dhabi for the kind hospitality during part of this work and Milvia Capalbi for her precious support during the analysis of some XRT observations. We thank Phil Evans for useful discussions. A.P. is supported by a Juan de la Cierva fellowship, and acknowledges grants AYA2012-39303, SGR2009-811, and iLINK2011-0303. P.R. acknowledges contract ASI-INAF I/004/11/0. We thank N. Schartel and the $X M M-N e w t o n$ team for having promptly performed the ToO observation analyzed in this paper. We are indebted to the Swift PI and operations team for the continuous support during the monitoring campaign of X-ray transients.

\section{References}

Campana, S., Coti Zelati, F., \& D’Avanzo, P. 2013, MNRAS, 432, 1695 Chenevez, J., Falanga, M., Brandt, S., et al. 2006, A\&A, 449, L5 Chenevez, J., Falanga, M., Kuulkers, E., et al. 2007, A\&A, 469, L27 Cocchi, M., Bazzano, A., Natalucci, L., et al. 2001, A\&A, 378, L37 Courvoisier, T., Walter, R., Beckmann, V., et al. 2003, A\&A, 411, L53 Cumming, A., \& Macbeth, J. 2004, ApJ, 603, L37

Evans, P. A., Beardmore, A. P., Page, K. L., et al. 2009, MNRAS, 397, 1177 Evans, P. A., Osborne, J. P., Beardmore, A. P., et al. 2014, ApJS, 210, 8 Falanga, M., Bonnet-Bidaud, J. M., Poutanen, J., et al. 2005a, A\&A, 436, 647 Falanga, M., Kuiper, L., Poutanen, J., et al. 2005b, A\&A, 444, 15 Falanga, M., Poutanen, J., Bonning, E. W., et al. 2007, A\&A, 464, 1069 Falanga, M., Chenevez, J., Cumming, A., et al. 2008, A\&A, 484, 43 Falanga, M., Cumming, A., Bozzo, E., \& Chenevez, J. 2009, A\&A, 496, 333 Falanga, M., Kuiper, L., Poutanen, J., et al. 2011, A\&A, 529, A68 Falanga, M., Kuiper, L., Poutanen, J., et al. 2012, A\&A, 545, A26 Ferrigno, C., Bozzo, E., Papitto, A., et al. 2014, A\&A, 567, A77

Fujimoto, M. Y., Sztajno, M., Lewin, W. H. G., \& van Paradijs, J. 1987, ApJ, 319,902

Fushiki, I., \& Lamb, D. Q. 1987, ApJ, 323, L55

Galloway, D. K., \& Cumming, A. 2006, ApJ, 652, 559

Galloway, D. K., Cumming, A., Kuulkers, E., et al. 2004, ApJ, 601, 466

Gierliński, M., \& Zdziarski, A. A. 2005, MNRAS, 363, 1349

Gilfanov, M., Revnivtsev, M., Sunyaev, R., \& Churazov, E. 1998, A\&A, 338, L83

Hartman, J. M., Galloway, D. K., \& Chakrabarty, D. 2011, ApJ, 726, 26

Heinke, C. O., Bahramian, A., Degenaar, N., \& Wijnands, R. 2015, MNRAS, 447, 3034 
Illarionov, A. F., \& Sunyaev, R. A. 1975, A\&A, 39, 185

in't Zand, J. J. M., Cumming, A., van der Sluys, M. V., Verbunt, F., \& Pols, O. R. 2005, A\&A, 441, 675

Jansen, F., Lumb, D., Altieri, B., et al. 2001, A\&A, 365, L1

Kennea, J. A., Burrows, D. N., Cummings, J. R., et al. 2013, ATel, 5354, 1

King, A. R., \& Ritter, H. 1998, MNRAS, 293, L42

Krimm, H. A., Holland, S. T., Corbet, R. H. D., et al. 2013, ApJS, 209, 14

Kuulkers, E., in't Zand, J. J. M., van Kerkwijk, M. H., et al. 2002, A\&A, 382, 503

Kuulkers, E., den Hartog, P. R., in't Zand, J. J. M., et al. 2003, A\&A, 399, 663

Kuulkers, E., in't Zand, J. J. M., \& Lasota, J.-P. 2009, A\&A, 503, 889

Kuulkers, E., Eckert, D., Ferrigno, C., et al. 2013, ATel, 5361, 1

La Parola, V., Segreto, A., Cusumano, G., \& Maselli, A. 2013, ATel, 5646, 1

Lebrun, F., Leray, J. P., Lavocat, P., et al. 2003, A\&A, 411, L141

Lewin, W. H. G., van Paradijs, J., \& Taam, R. E. 1993, Space Sci. Rev., 62, 223
Linares, M., Wijnands, R., van der Klis, M., et al. 2008, ApJ, 677, 515

Lund, N., Budtz-Jørgensen, C., Westergaard, N. J., et al. 2003, A\&A, 411, L231

Malesani, D., Kennea, J. A., Burrows, D. N., et al. 2013, GRB Coordinates Network, 15172, 1

Molkov, S., Revnivtsev, M., Lutovinov, A., \& Sunyaev, R. 2005, A\&A, 434, 1069

Papitto, A., di Salvo, T., Burderi, L., et al. 2007, MNRAS, 375, 971

Powell, C. R., Haswell, C. A., \& Falanga, M. 2007, MNRAS, 374, 466

Romano, P., Campana, S., Chincarini, G., et al. 2006, A\&A, 456, 917

Romanova, M. M., Lovelace, R. V. E., Bachetti, M., et al. 2014, Eur. Phys. J. Web Conf., 64, 5001

Strohmayer, T., \& Bildsten, L. 2006, in New views of thermonuclear bursts, eds. W. H. G. Lewin, \& M. van der Klis, 113

Ubertini, P., Lebrun, F., Di Cocco, G., et al. 2003, A\&A, 411, L131

Vaughan, B. A., van der Klis, M., Wood, K. S., et al. 1994, ApJ, 435, 362

Vaughan, S., Goad, M. R., Beardmore, A. P., et al. 2006, ApJ, 638, 920 